\title{
Cyclotron radiation and emission in graphene
}

\author{
Takahiro Morimoto, ${ }^{1}$ Yasuhiro Hatsugai, ${ }^{2,3}$ and Hideo Aoki ${ }^{1}$ \\ ${ }^{1}$ Department of Physics, University of Tokyo, Hongo, Tokyo 113-0033, Japan \\ ${ }^{2}$ Institute of Physics, University of Tsukuba, Tsukuba 305-8571, Japan \\ ${ }^{3}$ Department of Applied Physics, University of Tokyo, Hongo, Tokyo 113-8656, Japan
}

(Received 27 December 2007; published 27 August 2008)

\begin{abstract}
Peculiarity in the cyclotron radiation and emission in graphene is theoretically examined in terms of the optical conductivity and relaxation rates to propose that graphene in magnetic fields can be a candidate for realizing the Landau-level laser, proposed decades ago [H. Aoki, Appl. Phys. Lett. 48, 559 (1986)].
\end{abstract}

DOI: 10.1103/PhysRevB.78.073406

PACS number(s): 71.70.Di, 76.40.+b

\section{INTRODUCTION}

There has been an increasing fascination with the physics of graphene, a monolayer of graphite, as kicked off by the experimental discovery of an anomalous quantum Hall effect (QHE). ${ }^{1,2}$ The fascination comes from a condensed-matter realization of the massless Dirac-particle dispersion on lowenergy scales in the honeycomb lattice, ${ }^{1,3-6}$ which is behind all the peculiar properties of graphene. In magnetic fields this appears as unusual Landau levels, where (i) the Landau levels $\left(=\sqrt{n} \hbar \omega_{c}, n\right.$ : Landau index) are unevenly spaced, (ii) the cyclotron frequency $\omega_{c}=(2 e / c \hbar) v_{F} \sqrt{B}$ is proportional to $\sqrt{B}$ rather than to $B$, and (iii) there is an extra Landau level right at the massless Dirac point $(E=0)$, which is outside the Onsager's semiclassical quantization. ${ }^{7}$ While various transport measurements (as exemplified by the QHE) have been extensively done, optical properties have also begun to be measured. For example, Sadowski et al. ${ }^{8}$ have performed a Landau-level spectroscopy for a large graphene sample. Inter-Landau-level transitions are observed at multiple energies, which are due to a peculiar optical selection rule $(|n| \leftrightarrow|n| \pm 1$ as opposed to the usual $n \leftrightarrow n \pm 1)$, as well as to the uneven Landau levels.

Now, if we look at the QHE physics, cyclotron emission from the QHE system in nonequilibrium has been one important phenomenon. Experimentally, this typically appears as a strong cyclotron emission from the "hot spot:" a singular point in a Hall-bar sample where the convergence of electric lines of force puts the electrons out of equilibrium. ${ }^{9}$ For a bulk nonequilibrium effect, one of the present authors proposed theoretically a "Landau-level laser" for nonequilibrium QHE systems. ${ }^{10}$ The basic idea is simple enough: we can exploit the unusual coalescence of the energy spectrum into a series of line spectrum (Landau levels) to realize a laser from a spontaneous emission if we can make a population inversion, where the photon energy (=cyclotron energy in this case) is tunable and falls on the terahertz region for $B \sim 10 \mathrm{~T}$. However, the most difficult part is the population inversion, since if we, e.g., optically pump the system, the excitation would go up the ladder of equidistant Landau levels indefinitely.

This has motivated us to put a question (Fig. 1): will the graphene Landau levels, with uneven spacing among their peculiarities, favor in realizing such a population inversion? In this Brief Report, we show that this is indeed the case by actually calculating the optical conductivity as well as the relaxation processes. The message then is that graphene is a candidate for the Landau-level laser.

\section{OPTICAL CONDUCTIVITY IN GRAPHENE}

Low-energy physics around the Fermi energy in graphene is described by the massless Dirac Hamiltonian, ${ }^{4}$

$$
H_{0}=v_{F}\left(\begin{array}{cccc}
0 & \pi^{-} & 0 & 0 \\
\pi^{+} & 0 & 0 & 0 \\
0 & 0 & 0 & \pi^{+} \\
0 & 0 & \pi^{-} & 0
\end{array}\right),
$$

where $v_{F}$ is the velocity of the Dirac electron at $E_{F}, \pi^{ \pm}$ $\equiv \pi_{x} \pm i \pi_{y}$ with $\boldsymbol{\pi}=\mathbf{p}+e \mathbf{A}, \mathbf{A}$ is the vector potential representing a uniform magnetic field $\mathbf{B}=\operatorname{rot} \mathbf{A}$, and the $4 \times 4$ matrix is spanned by the chirality and $\left(K, K^{\prime}\right)$ Fermi points. In magnetic fields the energy spectrum is quantized into Landau levels,

$$
\begin{gathered}
\varepsilon_{n}=\operatorname{sgn}(n) \sqrt{n} \hbar \omega_{c}, \\
\omega_{c}=\frac{\sqrt{2}}{\ell} v_{F}=v_{F} \sqrt{\frac{2 e B}{\hbar},}
\end{gathered}
$$

for a clean system, where $n=0, \pm 1, \ldots$ is the Landau index and $\ell=\sqrt{\hbar / e B}$ is the magnetic length. Here we consider realistic systems having a disorder with the self-consistent Born approximation (SCBA) introduced by $\mathrm{Ando}^{4,11}$ to calculate the optical conductivity.
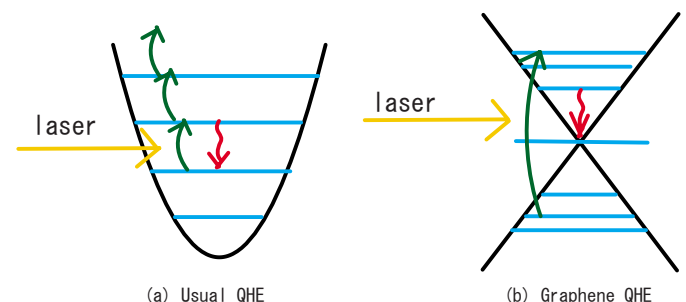

FIG. 1. (Color online) Cyclotron absorption (green) and emission (red) processes schematically depicted for the ordinary (a) and graphene (b) quantum Hall systems. Black lines represent the band dispersion, while blue lines the Landau levels. 


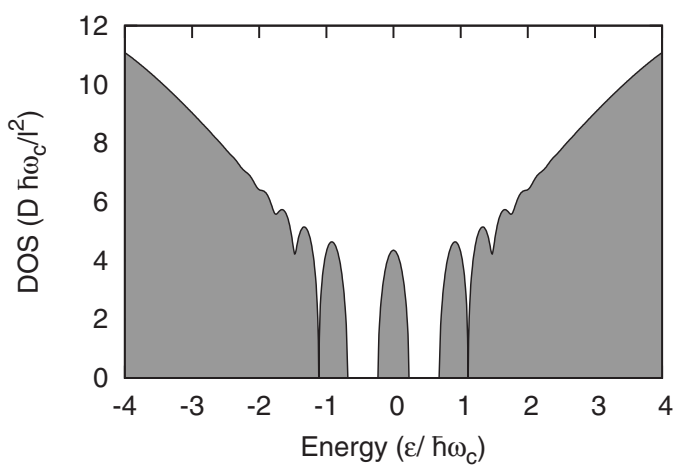

FIG. 2. A typical density of states for graphene for an intermediate disorder $\left(\Gamma / \omega_{c}=0.25\right.$ here $)$.

The optical conductivity is given by

$$
\begin{aligned}
\sigma_{\alpha \beta}(\omega)= & \frac{e^{2} \hbar}{i \pi} \int d \varepsilon \frac{f(\varepsilon)}{\hbar \omega} \\
& \times\left\{\operatorname{Tr}\left[j_{\alpha} \operatorname{Im} G(\varepsilon) j_{\beta}\left(G^{+}(\varepsilon+\hbar \omega)-G^{+}(\varepsilon)\right)\right]\right. \\
& \left.-\operatorname{Tr}\left[j_{\alpha}\left(G^{-}(\varepsilon)-G^{-}(\varepsilon-\hbar \omega)\right) j_{\beta} \operatorname{Im} G(\varepsilon)\right]\right\},
\end{aligned}
$$

where $\alpha, \beta=x, y, f(\varepsilon)$ as the Fermi distribution, and $G^{ \pm}$ $=G(\epsilon \pm i \delta)$ with a positive infinitesimal $\delta$. For Green's function $G$, with the self-energy $\Sigma_{n}(\varepsilon)=\frac{\Gamma^{2}}{4} \Sigma_{n^{\prime}}\left[\varepsilon-\operatorname{sgn}\left(n^{\prime}\right) \sqrt{\left|n^{\prime}\right|}\right.$ $\left.-\Sigma_{n}(\varepsilon)\right]^{-1}$ in the SCBA, the Landau-level broadening is given by $\frac{\Gamma^{2}}{4}=n_{0} V_{0}^{2} / 4 \pi \ell^{2}$ if we assume (for simplicity) a random potential arising from short-ranged scatterers $V$ $=\sum_{i} V_{0} \delta\left(\mathbf{r}-\mathbf{r}_{i}\right)$. The light absorption rate is then related to the imaginary part of the dielectric function $\varepsilon(\omega)=1$ $+i \sigma_{x x}(\omega) / \varepsilon_{0} \omega$ so we can look at $\operatorname{Re} \sigma_{x x}(\omega)$.

In order to discuss the optical conductivity in graphene, we need the current matrix elements across Landau levels. The eigenfunctions of the Hamiltonian (1) dictate an unusual selection rule $|n|-\left|n^{\prime}\right|= \pm 1$ in place of the ordinary $n-n^{\prime}$ $= \pm 1$ with $^{11}$

$$
\begin{aligned}
& j_{x}^{n, n^{\prime}}=v_{F} C_{n} C_{n^{\prime}}\left[\operatorname{sgn}(n) \delta_{|n|-1,\left|n^{\prime}\right|}+\operatorname{sgn}\left(n^{\prime}\right) \delta_{|n|+1,\left|n^{\prime}\right|}\right], \\
& j_{y}^{n, n^{\prime}}=i v_{F} C_{n} C_{n^{\prime}}\left[\operatorname{sgn}(n) \delta_{|n|-1,\left|n^{\prime}\right|}-\operatorname{sgn}\left(n^{\prime}\right) \delta_{|n|+1,\left|n^{\prime}\right|}\right],
\end{aligned}
$$

where $C_{n}=1(n=0)$ or $1 / \sqrt{2}$ (otherwise).

We have numerically obtained the Green's function and the optical conductivity. While in usual cases the broadened Landau levels are simultaneously merged or separated as $\Gamma$ is varied, there is a striking difference for graphene, where the Landau levels $(\propto \sqrt{n})$ are unevenly spaced, so that the broadened Landau levels overlap to a lesser extent as we go to the central one $(|n| \rightarrow 0)$ as typically depicted in Fig. 2. Namely, for an intermeditate value of $\Gamma / \hbar \omega_{c}$, only the $n=0$ Landau level stands alone while the other levels form a continuous spectrum.

We now look at the result for the optical conductivity in Fig. 3 for the Fermi energy at $\varepsilon_{F}=0$ (energy for the Dirac point), where each resonance peak can be assigned to an allowed transition with the selection rule [Eq. (5)]. The largest peak around $\omega / \omega_{c}=1$ corresponds to the transition between $n=0 \leftrightarrow \pm 1$, while the peaks at higher frequencies

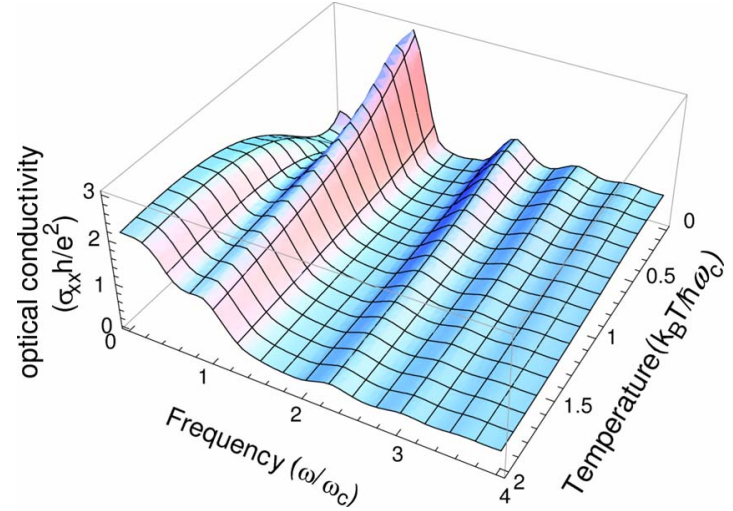

FIG. 3. (Color online) Optical conductivity against $\omega$ is shown for temperatures $k_{B} T / \hbar \omega_{c}=0-2.0$ for a fixed value of $\Gamma / \hbar \omega_{c}$ $=0.25$ with $\varepsilon_{F}=0$.

come from the transition across the Fermi energy $-n \leftrightarrow n \pm 1$. If we turn to the temperature dependence in the figure, we notice a peculiar phenomenon: there is a peak in the region $\omega / \omega_{c}<1$ that grows, rather than decays, for higher $T$. We can identify this as coming from the unusual Landau levels in graphene: as $T$ is raised with the Fermi distribution function (becoming longer tailed) higher Landau levels begin to be occupied, which enables the transitions among higher Landau levels $n \leftrightarrow n \pm 1$ to take place. While this would not cause other lines to appear for equidistant Landau levels, this does so for the unequally spaced Landau levels $(\propto \sqrt{|n|})$ for $\omega / \omega_{c}<1$ transitions. So we can identify this property as one hallmark of the "massless Dirac" dispersion.

Previously, the optical conductivity has been obtained by Gusynin et al., ${ }^{12,13}$ who have derived the analytical expression for the optical conductivity, but the self-energy from the disorder was set to a constant while we have calculated the self-energy self-consistently with SCBA. Sadowski et al. ${ }^{8}$ also presented a similar expression for the conductivity with a constant self-energy as well. The present result qualitatively agrees with these results, but the findings here are, first, the full dependence on the $k_{B} T / \hbar \omega_{c}$, including the growing of low-frequency peaks at low temperatures. Second, we point out that the situation as depicted in Fig. 2 should be interesting for the cyclotron resonance and emission in nonequilibrium situations induced by, e.g., an optical pumping with laser beams. Namely, the electrons excited to higher energies will relax down to the $n=1$ level across the continuum spectrum, so that the population inversion across $n=0$ and $n \geq 1$ should be easier to be realized.

\section{RELAXATION PROCESSES}

To quantify the above idea, we have to consider the relaxation processes which should control the population inversion. For the ordinary quantum Hall systems, the relaxation processes have been extensively discussed. Specifically, Chaubet et al. ${ }^{14,15}$ discussed dissipation mechanisms, where spontaneous photon radiation and coupling with phonons are examined on the basis of Fermi's golden rule. Other dissipation processes such as electron-electron or impurity scatter- 
ings, which conserve the total energy, do not contribute to inter-Landau-level processes in the absence of external electric fields (while Chaubet et al. have focused on effects of finite electric fields in the QHE breakdown where interLandau-level processes are involved).

So we extend the discussion by Chaubet et al. to relaxation processes in graphene. We first estimate the efficiency of the photon emission with Fermi's golden rule,

$$
W_{i \rightarrow f}=\frac{2 \pi}{\hbar}\left|\left\langle i\left|H_{\mathrm{int}}\right| f\right\rangle\right|^{2} \delta\left(\epsilon_{f}-\epsilon_{i}\right) .
$$

Here $|i\rangle\left(\epsilon_{i}\right)$ is the wave function (energy) in the initial state while $f$ stands for the final states and $H_{\text {int }}$ is the interaction Hamiltonian between the electromagnetic field and electrons,

$$
H_{\text {int }}=e \sum_{\lambda} \sqrt{\frac{\hbar}{2 \epsilon_{0} V \omega_{\lambda}}}\left[e^{i \mathbf{k} \cdot \mathbf{r}}\left(\mathbf{e}_{\lambda} \cdot \mathbf{v}\right) a_{\lambda}+e^{-i \mathbf{k} \cdot \mathbf{r}}\left(\mathbf{e}_{\lambda} \cdot \mathbf{v}\right) a_{\lambda}^{\dagger}\right],
$$

where $\epsilon_{0}$ is the permittivity of the vacuum, $V$ is the volume of the space where photons exist, $\omega_{\lambda}$ is the frequency of the light with mode $\lambda$ and the wave number $\mathbf{k}, \mathbf{e}_{\lambda}$ is the polarization vector, $a_{\lambda}$ is the annihilation operator of light, and $\mathbf{v}$ is the velocity of electrons.

When the wavelength of light is much larger than the cyclotron radius, as is usually the case, we can drop the $e^{i \mathbf{k} \cdot \mathbf{r}}$ term and we have

$$
\begin{aligned}
W_{i \rightarrow f} & =\frac{2 \pi}{\hbar} \frac{V}{\pi^{2} c^{3}} \int \omega^{\prime 2} d \omega^{\prime} \frac{e^{2} \hbar}{2 \epsilon_{0} V \omega^{\prime}}|\langle i|v| f\rangle|^{2} \delta\left(\hbar \omega^{\prime}+\epsilon_{f}-\epsilon_{i}\right) \\
& =4 \alpha\left(\frac{|\langle i|v| f\rangle|}{c}\right)^{2} \omega
\end{aligned}
$$

where $c$ is the velocity of light, $\alpha=e^{2} /\left(4 \pi \epsilon_{0} \hbar c\right)$ is the finestructure constant, and we put $\hbar \omega=\epsilon_{f}-\epsilon_{i}$ to be the cyclotron energy $\hbar \omega_{c}$.

A peculiarity of graphene appears in the current matrix element [Eq. (5)], for which the rate of the spontaneous emission, with $|\langle n|v| n+1\rangle|=C_{n} C_{n+1} v_{F}$ for graphene plugged in, reads

$$
W_{n+1 \rightarrow n}^{\text {graphene }}=\left\{\begin{array}{cc}
2 \alpha\left(\frac{v_{F}}{c}\right)^{2} \omega & (n=0), \\
\alpha\left(\frac{v_{F}}{c}\right)^{2} \omega & (n \neq 0) .
\end{array}\right.
$$

This expression, another key result here, shows that the spontaneous emission rate depends linearly on the cyclotron energy and quadratically on the Fermi velocity. This is in sharp contrast to the ordinary QHE systems such as the twodimensional electron gas (2DEG) realized at, e.g., GaAs/ AlGaAs, interfaces. In this case the velocity matrix element $|\langle n|v| n+1\rangle|^{2}=(n+1) \hbar \omega / 2 m^{*}$ should be plugged in Eq. (7), which yields

$$
W_{n+1 \rightarrow n}^{\mathrm{GaAs}}=2(n+1) \alpha \frac{\hbar}{m^{*} c^{2}} \omega^{2} .
$$

This reveals a dramatic difference between graphene and usual 2DEG, where the emission rate in the latter is proportional to the square of the cyclotron energy.

We can quantitatively realize the difference: the cyclotron energies are

$$
\hbar \omega= \begin{cases}\hbar e B / m^{*} \sim 1.7 \mathrm{meV} & \text { (GaAs) } \\ v_{F} \sqrt{2 \hbar e B} \simeq 37 \mathrm{meV} & \text { (graphene) }\end{cases}
$$

where on the right-hand sides we put $B=1 \mathrm{~T}$, adopted the value of graphene Fermi velocity $v_{F}=1.06 \times 10^{6} \mathrm{~m} / \mathrm{s},{ }^{8}$ and the GaAs effective mass $m^{*} \simeq 0.067 m_{e}$. Hence the cyclotron energy in graphene is an order of magnitude larger, which reflects $\omega_{c} \propto \sqrt{B}$ for the Dirac dispersion, while the energy is usually proportional to $B$. If we plug these in Eqs. (8) and (9), we end up with

$$
W_{i \rightarrow f}\left\{\begin{array}{lll}
\propto B^{2} \simeq 6 \times 10^{4} & \left(\mathrm{~s}^{-1}\right) & (\mathrm{GaAs}) \\
\propto \sqrt{B} \simeq 1 \times 10^{7} & \left(\mathrm{~s}^{-1}\right) & \text { (graphene) }
\end{array}\right.
$$

where the right-hand sides are for $B=1$ T. A conspicuous difference, $\propto B^{2}$ in the former and $\propto \sqrt{B}$, should sharply affect the behavior and the spontaneous photon emission rate is orders of magnitude enhanced in graphene in moderate magnetic fields (as in the above numbers quoted for $B=1 \mathrm{~T}$.) This indicates that the present system is indeed favorable for a realization of the envisaged Landau-level laser.

Now, the dissipation process which competes with the photon emission is the phonon emission process, which has been discussed for the conventional QHE systems, in the context of the breakdown of the QHE. ${ }^{14}$ The phonon emission rate is also obtained from Fermi's golden rule if we replace the electron-light interaction with the electronphonon interaction. If we first consider acoustic phonons, the dissipation rate is proportional to the extent of the overlap between initial and final wave functions both in usual and graphene QHE systems, which yields a factor $e^{-(q \ell)^{2}}$, with $q$ the phonon wave number and $\ell$ the magnetic length. In usual QHE systems the cyclotron energy is $\sim 1 \mathrm{meV}$ and the magnetic length $\ell=\sqrt{\hbar / e B} \sim 30 \mathrm{~nm}$ for $B=1 \mathrm{~T}$, while the acoustic-phonon wave number is $\sim 1 \AA^{-1}$, so that the overlap factor is exponentially small. The situation is similar in graphene since the magnetic length $\ell=\sqrt{\hbar / e B}$ is the same. So the acoustic-phonon emission should be negligible in graphene as well as in weak electric fields. When the applied laser electric field is so intense $(\sim 1 \mathrm{kV} / \mathrm{cm})$ that the Landau levels are distorted and the overlap factor grows, the phonon emission may begin to compete with the photoemission.

Are there any other factors that distinguish graphene from 2DEGs? In this context, we can note that Chaubet et al. have further pointed out the following. In an electron system confined to a $2 \mathrm{D}$, a wave function has a finite tail in the direction normal to the plane and the phonon emission is enhanced through the coupling of the tail of the wave function and perpendicular phonon modes, which propagate normal to the $2 \mathrm{D}$ system in the substrate. ${ }^{15}$ In this way the phonon emission can compete with the spontaneous emission in usual 
QHE systems. In contrast, a graphene sheet is an atomic monolayer with only a loose coupling with the substrate. We can also consider a combined effect of scattering of electrons by acoustic phonons and impurity scattering, which may compensate for the momentum transfer $q$ of phonons, and hence the overlap factor $e^{-(q \ell)^{2}} \cdot{ }^{16,21}$ To be precise, graphene itself should have phonon modes that include the out-ofplane modes and their effects are interesting future problems. As for optical phonons, their energies are known to be higher than $100 \mathrm{meV}$ for $q \simeq 0$ in graphene ${ }^{17}$ so that optical phonons do not contribute to the dissipation for $B \sim$ a few tesla with $\hbar \omega \sim 40 \mathrm{meV}$. Overall, we conclude that the dissipation due to acoustic phonons will be small in graphene in the weak electric-field regime. When the pumping laser intensity is not too strong to invalidate the present treatment but strong enough for the population inversion, the present reasoning should apply and we can expect efficient cyclotron emissions from graphene.

Entirely different, but interesting, is the problem of Anderson localization arising from the disorder. While this is out of scope of the present work, we can expect delocalized states, with diverging localization length at the center of each Landau level, are present as inferred from the QHE observation and this poses an interesting future problem. The situation should also depend on whether the disorder is short range or long range; but, in ordinary QHE systems, a sum rule guarantees the total intensity of the cyclotron resonance intact. ${ }^{10}$ As for the "ripples" (known to exist in actual graphene samples ${ }^{18}$ ), the $n=0$ Landau level remains sharp (which is topologically protected since the slowly varying potential does not destroy the chiral symmetry ${ }^{19}$ ) while other levels become broadened ${ }^{20}$ and this should favor the situation proposed in the presented Brief Report. ${ }^{22}$

\section{SUMMARY}

To summarize, we have discussed the cyclotron radiation from graphene QHE system. We conclude that unusual uneven Landau levels, unusual cyclotron energy, and unusual transition selection rules in graphene all work favorably for a population inversion envisaged for the Landau-level laser. An estimate of the photon emission rate shows that the emission rate is of the orders of magnitude more efficient than in the ordinary QHE system, while the competing phonon emission rate is not too large to mar the photon emission. Important future problems include the examination of the actual lasing processes including the cavity properties, coupling of electrons to the out-of-plane phonon modes, etc.

\section{ACKNOWLEDGMENTS}

We wish to thank Andre Geim for his illuminating discussions. This work has been supported in part by Grants-in-Aid for Scientific Research on Priority Areas from MEXT, "Physics of new quantum phases in superclean materials" (Grant No. 18043007) for Y.H., and "Anomalous quantum materials" (Grant No. 16076203) for H.A.
${ }^{1}$ K. Novoselov, A. Geim, S. Morozov, D. Jiang, M. Katsnelson, I. Grigorieva, S. Dubonos, and A. Firsov, Nature (London) 438, 197 (2005).

${ }^{2}$ Y. Zhang, Y. W. Tan, H. L. Stormer, and P. Kim, Nature (London) 438, 201 (2005).

${ }^{3}$ J. McClure, Phys. Rev. 104, 666 (1956).

${ }^{4}$ Y. Zheng and T. Ando, Phys. Rev. B 65, 245420 (2002).

${ }^{5}$ V. P. Gusynin and S. G. Sharapov, Phys. Rev. Lett. 95, 146801 (2005).

${ }^{6}$ N. M. R. Peres, F. Guinea, and A. H. Castro Neto, Phys. Rev. B 73, 125411 (2006).

${ }^{7}$ L. Onsager, Philos. Mag. 43, 1006 (1952).

${ }^{8}$ M. L. Sadowski, G. Martinez, M. Potemski, C. Berger, and W. A. de Heer, Phys. Rev. Lett. 97, 266405 (2006).

${ }^{9}$ K. Ikushima, H. Sakuma, S. Komiyama, and K. Hirakawa, Phys. Rev. Lett. 93, 146804 (2004).

${ }^{10}$ H. Aoki, Appl. Phys. Lett. 48, 559 (1986).

${ }^{11}$ T. Ando, J. Phys. Soc. Jpn. 38, 989 (1975).

${ }^{12}$ V. P. Gusynin, S. G. Sharapov, and J. P. Carbotte, Phys. Rev. Lett. 98, 157402 (2007).

${ }^{13}$ V. P. Gusynin and S. G. Sharapov, Phys. Rev. B 73, 245411
(2006).

${ }^{14}$ C. Chaubet, A. Raymond, and D. Dur, Phys. Rev. B 52, 11178 (1995).

${ }^{15}$ C. Chaubet and F. Geniet, Phys. Rev. B 58, 13015 (1998).

${ }^{16}$ Localized states around point defects were detected recently with STM in $B$ (Ref. 21) and their radius was found to be comparable to the magnetic length $(\sim 30 \mathrm{~nm})$.

${ }^{17}$ S. Piscanec, M. Lazzeri, F. Mauri, A. C. Ferrari, and J. Robertson, Phys. Rev. Lett. 93, 185503 (2004).

${ }^{18}$ J. Meyer, A. Geim, M. Katsnelson, K. Novoselov, T. Booth, and S. Roth, Nature (London) 446, 60 (2007).

${ }^{19}$ Y. Hatsugai, T. Fukui, and H. Aoki, Phys. Rev. B 74, 205414 (2006).

${ }^{20}$ A. Giesbers, U. Zeitler, M. Katsnelson, L. Ponomarenko, T. Mohiuddin, and J. Maan, Phys. Rev. Lett. 99, 206803 (2007).

${ }^{21}$ Y. Niimi, H. Kambara, T. Matsui, D. Yoshioka, and H. Fukuyama, Phys. Rev. Lett. 97, 236804 (2006).

${ }^{22}$ We can also note that the $n=0$ Landau level in graphene has an exactly $E=0$ edge mode, of a topological origin, right at the center (Ref. 19), which may also affect the photon absorption/ emission. 